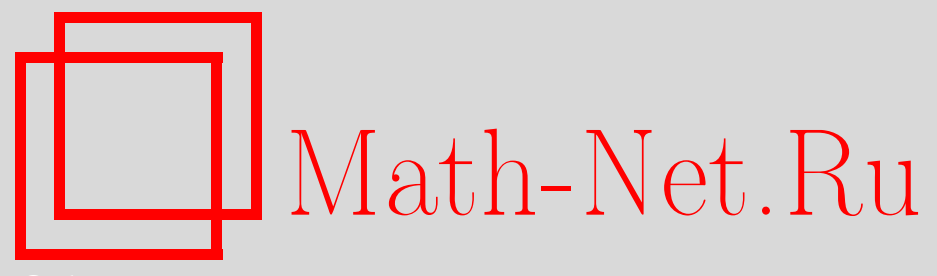

Н. Ю. Демин, О необходимом количестве правил автоматной грамматики, порождающей конечный язык, Дискрет. матем., 2000, том 12, выпуск 4, 99-108

DOI: https://doi.org/10.4213/dm350

Использование Общероссийского математического портала Math-Net.Ru подразумевает, что вы прочитали и согласны с пользовательским соглашением http://www . mathnet.ru/rus/agreement

Параметры загрузки:

IP : 3.85 .5 .30

26 апреля 2023 г., 17:09:16

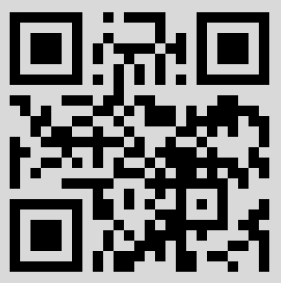




\title{
Аискретная математика
}

том 12 выпуск $4 * 2000$

Удк 519.7

\section{О необходимом количестве правил автоматной грамматики, порождающей конечный язык}

\author{
(C) 2000 г. Н. Ю. Демин
}

\begin{abstract}
Рассматривается проблема восстановления протокола передачи данных по обмену его сообщениями. Формально данную проблему можно свести к задаче синтеза грамматики по порождаемому ею языку. Приводится оценка необходимого числа правил в автоматной грамматике, которая порождает язык данной конечной мощности.
\end{abstract}

\section{1. Введение}

В настоящее время в области информационных технологий приходится сталкиваться с проблемой изучения реально применяемых протоколов передачи данных, которые не соответствуют своим стандартам. В большинстве случаев такая проблема возникает в процессе интеграции различного телекоммуникационного оборудования.

Обычно под протоколом передачи данных, сетевым протоколом или просто протоколом понимают формат описания передаваемых сообщений и правила, по которым происходит обмен информацией между двумя или несколькими системами. Следовательно, естественно рассматривать протокол как формальную грамматику $\Gamma$ (см. [1]), порождающую некоторое множество сообщений - язык $\mathscr{L}(\Gamma)$. Таким образом, задача восстановления протокола по обмену его сообщениями сводится к задаче синтеза грамматики по порождаемому ею языку. Другими словами, дан язык $L$ и необходимо построить такую грамматику $\Gamma$, что порожденный ею язык $\mathscr{L}(\Gamma) \supseteq L$. В качестве меры сложности восстанавливаемой грамматики берется ее мощность, то есть число ее правил.

В статье приводится порядок величины, равной минимальной мощности автоматной грамматики (см. [2]), порождающей с шумом фиксированное количество слов. Автор выражает благодарность Э. Э. Гасанову и Э. А. Применко за постановку задачи и помощь в работе.

\section{2. Основные понятия и результаты}

Пусть $A-$ множество символов, называемое алфавитом. Слово $\delta$ в алфавите $A-$ это последовательность символов

$$
\delta=a_{1} a_{2} \ldots a_{n}, \quad a_{1}, a_{2}, \ldots, a_{n} \in A .
$$


Обозначим $A^{*}$ множество всех слов конечной длины в алфавите $A$.

Языком $L$ над алфавитом $A$ называется произвольное подмножество множества $A^{*}$.

Грамматика - это упорядоченная четверка $\Gamma=\left(W_{T}, W_{N}, I, R\right)$, где $W_{T}$ - конечный основной (терминальный) алфавит, $W_{N}$ - конечный вспомогательный (нетерминальный) алфавит, $I \in W_{N}-$ начальный символ грамматики $\Gamma, R-$ конечное множество правил $\left\{u_{i} \rightarrow v_{i} \mid i=1,2, \ldots, k\right\}$ (конечная система подстановок), левые и правые части которых есть слова $u_{i}, v_{i} \in\left(W_{T} \cup W_{N}\right)^{*}$, причем символ $\rightarrow \notin\left(W_{T} \cup W_{N}\right)^{*}$.

Будем говорить, что слово $y$ непосредственно выводится из слова $x$ в грамматике $\Gamma$ и обозначать $x \Rightarrow y$, если существует правило $u \rightarrow v \in R$ такое, что $x=x_{1} u x_{2}$ и $y=x_{1} v x_{2}$, где $x_{1}, x_{2} \in\left(W_{T} \cup W_{N}\right)^{*}$.

Слово $y$ называется выводимым из слова $x$ в грамматике $\Gamma$, что обозначается $x \stackrel{*}{\Rightarrow}$ $y$, если слова $x, y$ совпадают или существует последовательность слов $z_{0}, z_{1}, \ldots, z_{k}$ такая, что

$$
z_{0}=x, \quad z_{k}=y, \quad z_{i-1} \Rightarrow z_{i}, \quad i=1, \ldots, k
$$

Последовательность $D=\left(z_{0}, z_{1}, \ldots, z_{k}\right)$, где $z_{0}=x$ и $z_{k}=y$, называется выводом слова $y$ из слова $x$ в грамматике $\Gamma$. Вывод $D$ в грамматике называется полным, если $y=z_{k} \in W_{T}^{*}$. Слово $y \in W_{T}^{*}$ называется правильным, если существует по крайней мере один полный вывод слова $y$ из слова $x=I$.

Язык $\mathscr{L}(\Gamma)$, порожденный грамматикой $\Gamma,-$ это множество всех правильных слов в грамматике $\Gamma$.

Автоматная грамматика - это такая грамматика $\Gamma$, каждое правило которой имеет вид $u \rightarrow z$, где $z=x$ или $z=x \tau$ и $x \in W_{T}, u, \tau \in W_{N}$.

Грамматику $Г$ назовем ациклической, если она порождает конечный язык.

Нетерминальный символ назовем несущественным, если он не встречается ни в одном полном выводе из начального символа $I$.

В дальнейшем будем рассматривать множество $A_{2}$ - множество всех автоматных грамматик, не содержащих несущественных нетерминальных символов с $W_{T}=$ $\{0,1\}$. Под мощностью грамматик $\Gamma \in A_{2}$ будем понимать число ее правил и обозначать это число $|\Gamma|$.

Пусть $A_{2}(n)$ - множество ациклических автоматных грамматик из $A_{2}$ с числом правил, не большим $n$. Обозначим через $F(n)$ максимальное число слов, которое можно породить ациклической грамматикой из $A_{2}(n)$, то есть

$$
F(n)=\max _{\Gamma \in A_{2}(n)}|\mathscr{L}(\Gamma)|
$$

Теорема 1. Для любого натуралъного $n$

$$
F(n)= \begin{cases}2^{l}, & \text { если } n=2 l \\ 2^{l}+\left[2^{l-2}\right], & \text { если } n=2 l+1 .\end{cases}
$$

Пусть $A(L)$ - множество всех грамматик из $A_{2}$, порождающих язык $L$, и

$$
M_{0}(m)=\min _{|\mathscr{L}(\Gamma)|=m}|\Gamma|
$$


Пусть даны язык $L$ и грамматика $Г$ из $A_{2}$ такая, что $\mathscr{L}(\Gamma) \supseteq L$. Уровнем шума грамматики $\Gamma$ по отношению к языку $L$ назовем величину

$$
N(\Gamma, L)=\frac{|\mathscr{L}(\Gamma) \backslash L|}{|L|}
$$

Обозначим через $A_{c}(L)$ множество всех грамматик с уровнем шума $N(\Gamma, L) \leqslant c$ по отношению к языку $L$, а через $\Gamma^{c}(L)$ - грамматику, принадлежащую $A_{c}(L)$ такую, что

$$
\left|\Gamma^{c}(L)\right|=\min _{\Gamma \in A_{c}(L)}|\Gamma|
$$

Пусть

$$
M_{c}(m)=\min _{|L|=m}\left|\Gamma^{c}(L)\right|,
$$

а $Q(m)$ - функция, определенная равенствами

$$
Q(m)= \begin{cases}2, & \text { если } m=2, \\ 4, & \text { если } m=3,4 \\ 2 k+1, & \text { если } 2^{k}+1 \leqslant m \leqslant 2^{k}+2^{k-2}, k>1, \\ 2 k+2, & \text { если } 2^{k}+2^{k-2}+1 \leqslant m \leqslant 2^{k+1}, k>1 .\end{cases}
$$

Теорема 2. Пусть $m$ - натуральное число,

$$
m=\sum_{i=0}^{k} a_{i} 2^{i}
$$

тогда

$$
Q(m) \leqslant M_{0}(m) \leqslant 2 k+2 a_{k-1}+\sum_{i=0}^{k-2} a_{i} .
$$

Теорема 3. Для любого натуралъного $m>1 u c \geqslant 3 / 5$

$$
M_{c}(m)=Q(m)
$$

\section{3. Оценка максимальной мощности конечного языка, порождаемого конечной грамматикой}

Пусть дан ориентированный граф $G=\{V, X\}$, где $V$ - множество его вершин и $X$ - множество его ребер. Начальная и конечная вершины графа $G$ - это вершина, в которую не входит и из которой не выходит ни одного ребра соответственно. Граф $G$ назовем правильным графом, если у него одна начальная и одна конечная вершины, любое ребро имеет кратность, не большую 2, и в графе нет ориентированных циклов. Обозначим через $C(G)$ число ориентированных цепочек, которые начинаются в начальной и заканчиваются в конечной вершинах правильного графа $G$. Обозначим через $\Omega(n)$ множество правильных графов с $n$ ребрами. Пусть

$$
C(n)=\max _{G \in \Omega(n)} C(G)
$$


Pис. 1.
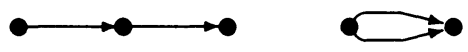

Pис. 2.

Пусть $n$ - натуральное число. Определим функцию $f(n)$ следующим образом:

$$
f(n)= \begin{cases}2^{l}, & \text { если } n=2 l \\ 2^{l}+\left[2^{l-2}\right], & \text { если } n=2 l+1\end{cases}
$$

Лемма 1. Для любого натуралъного $n$

$$
C(n)=f(n)
$$

Доказательство. Докажем индукцией по $n$, что $C(n) \leqslant f(n)$. В качестве базы индукции возьмем случай $n=1$. Если $n=1$, то $C(1)=1=f(1)$, поскольку существует только один правильный граф с одним ребром (рис. 1).

Если $n=2$, то $C(2)=2=f(2)$, поскольку существуют только два правильных графа с двумя ребрами (рис. 2).

Проведем шаг индукции. Пусть для всех $q \leqslant n$ утверждение леммы выполняется. Возьмем произвольный правильный граф $G$ с $n \geqslant 2$ ребрами, где $\alpha-$ начальная вершина графа, а $\Lambda$ - конечная вершина графа (рис. 3). Пусть из вершины $\alpha$ исходит $k$ ребер в вершины $\beta_{1}, \beta_{2}, \ldots, \beta_{k}$, причем какие-то из этих вершин могут совпадать. Обозначим через $G_{i}$ граф, составленный из всех вершин и ребер, принадлежащих ориентированным цепям, ведущим из $\beta_{i}$ в $\Lambda, i=1,2, \ldots, k$.

Так как в вершину $\alpha$ не входит ни одно ребро, ребра $\left(\alpha, \beta_{i}\right)$ не принадлежат ни одному из графов $G_{i}, i=1,2, \ldots, k$.

Отсюда следует, что

$$
C(G)=\sum_{i=1}^{k} C\left(G_{i}\right) \leqslant k C(n-k) \leqslant k f(n-k) .
$$

Заметим, что $f(n-2) \leqslant f(n) / 2$ для всех $n \geqslant 2$.

Возможны два случая.

Пусть $k=2 r$. Тогда

$$
\begin{aligned}
k f(n-k) & \leqslant \frac{k}{2} f(n-k+2) \\
& \leqslant \frac{k}{4} f(n-k+4) \leqslant \ldots \leqslant \frac{k}{2^{r}} f(n) \leqslant f(n)
\end{aligned}
$$




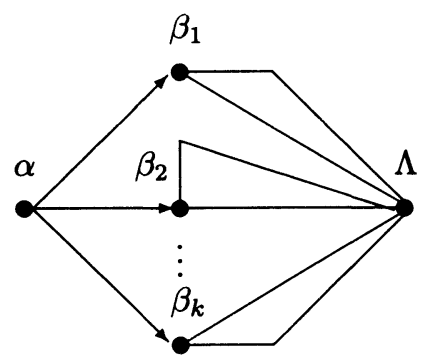

Рис. 3.

Пусть теперь $k=2 r+1$. Тогда

$$
\begin{aligned}
k f(n-k) & \leqslant \frac{k}{2} f(n-k+1) \\
& \leqslant \frac{k}{4} f(n-k+3) \leqslant \ldots \leqslant \frac{k}{2^{r}} f(n-1) \leqslant f(n) .
\end{aligned}
$$

Таким образом, для любого правильного графа $G$ с $n$ ребрами $C(G) \leqslant f(n)$. Следовательно, $C(n) \leqslant f(n)$.

Покажем, что $C(n) \geqslant f(n)$.

Возможны пять случаев.

Пусть $n=1$ и $G_{1}$ - правильный граф, изображенный на рис. 1 . В этом случае

$$
C(1) \geqslant C\left(G_{1}\right)=1=f(1) .
$$

Пусть $n=2$ и $G_{2}-$ правильный граф, изображенный на рис. 2. В этом случае

$$
C(2) \geqslant C\left(G_{2}\right)=2=f(2) .
$$

Пусть $n=3$ и $G_{3}-$ правильный граф, изображенный на рис. 4 . В этом случае

$$
C(3) \geqslant C\left(G_{3}\right)=2=f(3) .
$$

Пусть $n=2 l$, где $l>1$. Рассмотрим правильный граф $G_{2 l}$, изображенный на рис. 5. В этом случае

$$
C(n) \geqslant C\left(G_{2} l\right)=2^{l}=f(n) .
$$

Пусть $n=2 l+1$, где $l>1$. Рассмотрим правильный граф $G_{2 l+1}$, изображенный на рис. 6 . В этом случае

$$
C(n) \geqslant C\left(G_{2 l+1}\right)=2^{l}+2^{l-2}=f(n) .
$$

Таким образом, $C(n) \geqslant f(n)$. Отсюда следует, что $C(n)=f(n)$. Лемма доказана. 


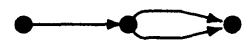

Pис. 4.

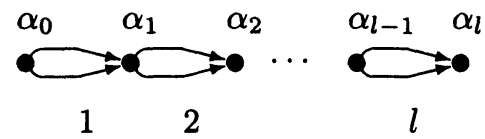

Pnc. 5.

Заметим, что любую автоматную грамматику

$$
\Gamma=\left(W_{T}, W_{N}, I, R\right)
$$

можно представить с помощью графа $G$, который строится следующим образом. Возьмем $\left|W_{N}\right|+1$ вершину, сопоставим одной из них символ $\Lambda \notin W_{N}$, а остальным взаимно однозначно сопоставим символы из $W_{N}$. Значок $\Lambda$ будет пониматься нами как отсутствие нетерминального символа в правой части правила, то есть правило вида $t \rightarrow a$ будем понимать как $t \rightarrow a \Lambda$. Из вершины $\alpha$ в вершину $\beta$ проведем ориентированное ребро в том и только том случае, когда в грамматике есть правило, левая и правая части которого содержат нетерминальные символы, соответствующие данным вершинам. Само ребро нагружается терминальным символом, входящим в правую часть данного правила. Полученный нагруженный ориентированный граф будем обозначать $G(\Gamma)$ и называть источником грамматики Г. Очевидно, что по источнику $G(\Gamma)$ однозначно восстанавливается грамматика $\Gamma$.

Вершину графа $G(\Gamma)$, которой сопоставлен начальный символ грамматики, назовем истоком, а вершину, которой приписан символ $\Lambda$, стоком.

Лемма 2. Любая цепочка в источнике $G(\Gamma)$ из начальной вериины в конечную соответствует некоторому слову, порождаемому данной грамматикой.

Доказательство. Пусть последовательность $v_{1} x_{1} v_{2} x_{2} v_{3} \ldots v_{k}$ - путь из начальной

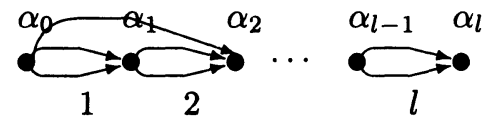

Рис. 6. 
вершины $v_{1}$ в конечную $v_{k}$. По определению $G(\Gamma)$

$$
v_{i} \rightarrow x_{i} v_{i+1} \in R, \quad i=1,2, \ldots, k-1,
$$

где $R \in \Gamma$, и при

$$
v_{1}=I, \ldots, v_{k}=\Lambda
$$

в грамматике Г существует вывод

$$
D=I \Rightarrow x_{1}, x_{1} \Rightarrow x_{1} x_{2}, \ldots, x_{1} x_{2} \ldots x_{k-2} \Rightarrow x_{1} x_{2} \ldots x_{k-1} \text {. }
$$

Лемма доказана.

Лемма 3. Если ациклическая грамматика $Г$ не содержит несущественных нетерминалъных символов $и|T|=2$, то источник $G(\Gamma)$ - правилъный граф.

Доказателъство. В силу того, что грамматика $\Gamma$ порождает конечное число слов, количество цепочек из начальной вершины графа $G(\Gamma)$ в вершину $\Lambda$ тоже конечно. В графе $G(\Gamma)$ нет ориентированных циклов, поскольку наличие хотя бы одного цикла приводит к бесконечному числу цепей, ведущих из начальной вершины в конечную, и следовательно, к бесконечному языку $L(G)$.

По определению $G(\Gamma)$ из вершины с символом $\Lambda$ ни одного ребра не выходит.

Докажем, что в исток ни одного ребра не заходит. Предположим, что в исток заходит ребро $(\alpha, I)$. Так как $\alpha$ - существенный нетерминал, он содержится в некотором выводе из начального символа, следовательно, мы имеем ориентированный цикл, проходящий через исток и приводящий к бесконечности языка $L(G)$. Получаем противоречие. Доказательство того, что из стока не исходит ни одно ребро, аналогично.

Докажем, что в графе $G(\Gamma)$ одна начальная и одна конечная вершины. По определению, вершина конечна, если из нее не выходит ни одного ребра. Предположим, что в графе $G(\Gamma)$ существует конечная вершина с символом $\alpha \neq \Lambda$. Но тогда через эту вершину не проходит ни одна цепочка из вершины $I$ в вершину $\Lambda$, следовательно, $\alpha$ - несущественный нетерминал. Получаем противоречие. Единственность начальной вершины доказывается аналогичным образом.

Кратность каждого ребра в $G(\Gamma)$ не больше, чем мощность алфавита, то есть не больше двух.

Лемма доказана.

\section{Следствие 1. Справедливо равенство}

$$
C(G(\Gamma))=|L(\Gamma)|
$$

Утверждение следует из лемм 2 и 3.

Лемма 4. Для любого правилъного графа существует такая нагрузка его вершин нетерминальными символами и нагрузка его ребер терминальными символами, что полученный нагруженный граф будет источником некоторой ациклической грамматики. 
Доказательство. Рассмотрим произвольный правильный граф $G$ с $\boldsymbol{n}$ вершинами. Возьмем множество $W_{N}$ такое, что $\left|W_{N}\right|=n-1$ и $W_{N}$ содержит $I$. Начальную вершину помечаем символом $I$, конечную - символом $\Lambda$, а остальные взаимно однозначно символами из $W_{N} \backslash I$. Нагружаются ребра символами 0,1 так, чтобы кратные ребра были нагружены разными символами. Каждому ребру $(\alpha, \beta)$ графа сопоставим правило $\mu \rightarrow a v$, где $a$ - символ, приписанный ребру, а $\mu$ и $v$ - символы, приписанные вершинам $\alpha$ и $\beta$ соответственно. Понятно, что правила, сопоставленные разным ребрам, разные. Пусть $R$ - множество правил, сопоставленных всем вершинам графа, то есть $|R|$ равно числу ребер в $G$. Очевидно, что полученный нагруженный граф будет источником для грамматики $\Gamma=\left(\{0,1\}, W_{N}, I, R\right)$.

Лемма доказана.

Докажем теперь теорему 1 . Утверждение теоремы следует из равенства

$$
\begin{aligned}
F(n) & =\max _{\Gamma \in A_{2}(n)}|L(\Gamma)|=\max _{G \in \Omega(n)} C(G) \\
& =f(n)= \begin{cases}2^{l}, & \text { если } n=2 l, \\
2^{l}+\left[2^{l-2}\right], & \text { если } n=2 l+1 .\end{cases}
\end{aligned}
$$

Теорема доказана.

\section{4. Оценка минимума мощности грамматик, порождающих с шумом заданное число слов}

Лемма 5. Если $l$ - натуралъное число, то $M_{0}\left(2^{l}\right)=2 l n p u l \geqslant 1 u M_{0}\left(2^{l}+2^{l-2}\right)=$ $2 l+1 n p u l \geqslant 2$.

Доказательство. Согласно теореме 1

$$
M_{0}\left(2^{l}\right)=M_{0}(F(2 l)) \leqslant 2 l .
$$

Предположим, что $M_{0}\left(2^{l}\right)=m<2 l$, то есть существует грамматика $\Gamma_{\min }$ такая, что

$$
\left|\Gamma_{\min }\right|=m, \quad\left|L\left(\Gamma_{\min }\right)\right|=2^{l} .
$$

Тогда

$$
F(m)=\max _{\Gamma \in A_{2}(m)}|L(\Gamma)| \geqslant\left|L\left(\Gamma_{\min }\right)\right|=2^{l} .
$$

Но так как $F(m)$ строго возрастает, $F(m)<F(2 l)$. Получаем противоречие. Доказательство утверждения для $M_{0}\left(2^{l}+2^{l-2}\right)$ аналогично.

Лемма доказана.

Лемма 6. Для любых натуральных $k \geqslant 2 u r$

$$
M_{0}\left(2^{k}+r\right) \geqslant 2 k+1, \quad M_{0}\left(2^{k}+2^{k-2}+r\right) \geqslant 2 k+2 .
$$

Доказательство. Покажем, что $M_{0}\left(2^{k}+r\right)>2 k$. Предположим противное, что $M_{0}\left(2^{k}+r\right)=m \leqslant 2 k$, то есть существует грамматика $\Gamma_{\min }$ такая, что

$$
\left|\Gamma_{\min }\right|=m, \quad\left|L\left(\Gamma_{\min }\right)\right|=2^{k}+r,
$$




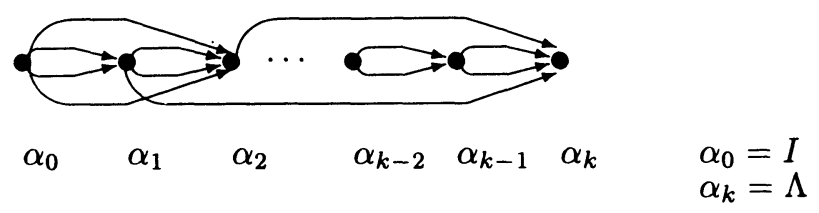

Рис. 7.

тогда

$$
F(m)=\max _{\Gamma \in A_{2}(m)}|L(\Gamma)| \geqslant\left|L\left(\Gamma_{\min }\right)\right|=2^{k}+r .
$$

Но $F(m) \leqslant F(2 k)=2^{k}$. Получаем противоречие. Для $M_{0}\left(2^{k}+2^{k-2}+r\right)$ доказательство аналогично.

Лемма доказана.

Доказателъство теоремъ 2. Сначала получим оценку сверху минимума мощности грамматик, порождающих заданное число слов. Пусть

$$
m=\sum_{i=0}^{k} a_{i} 2^{i}
$$

Построим $G(m)$ следующим образом. Возьмем граф, изображенный на рис. 5 , где $l=k$.

Если $a_{i}=1$, где $i<k-1$, то проводим ребро из вершины $\alpha_{i}$ в вершину $\alpha_{k}$. Это приводит к $2^{i}$ новым цепочкам, ведущим из вершин $\alpha_{0}$ в $\alpha_{k}$. Если $a_{k-1}=1$, то проводим два ребра из $\alpha_{0}$ в $\alpha_{2}$. Это приводит к $2 \cdot 2^{k-2}=2^{k-1}$ новым цепочкам из $\alpha_{0}$ в $\alpha_{k}$. Пример графа $G(m)$ приведен на рис. 7. Тем самым граф $G(m)$ содержит

$$
m=\sum_{i=1}^{k} a 2^{i}
$$

цепочек, ведущих из $\alpha_{0}$ в $\alpha_{k}$, или, что тоже самое, грамматика $\Gamma$, источником которой является $G(m)$, порождает $m$ слов. Следовательно,

$$
M_{0}(m)=\min _{|\mathscr{L}(\Gamma)|=m}|\Gamma| \leqslant\left|\Gamma_{m}\right|=2 k+2 a_{k-1}+a_{k-2}+\ldots+a_{0} .
$$

В силу лемм 5 и 6 условие $Q(m) \leqslant M_{0}(m)$ выполняется для любых $m \neq 3$. Покажем, что $M_{0}(3)=4$. Предположим противное, что $M_{0}(3)=3$, то есть существует грамматика Г такая, что

$$
|\Gamma|=3, \quad|L(\Gamma)|=3 .
$$

Тогда $F(3)=3$, но $F(3)=2$. Поэтому $M_{0}(3)=4$.

Теорема доказана.

Следствие 2. Для любого натуралъного $m>3$

$$
\text { 2] } \log _{2} m\left[\leqslant M_{0}(m) \leqslant 3\left[\log _{2} m\right]\right. \text {. }
$$


Справедливость следствия вытекает из леммы 6 и теоремы 2.

Дохазательство теоремы 3. Пусть $m$ - натуральное число и $m \geqslant 2$. Если $L-$ язык такой, что $|L|=m$, а $\Gamma \in R_{c}(L)$ - такая грамматика, что $\mathscr{L}(\Gamma) \geqslant L$, то из условия

$$
N(\Gamma, L)=\frac{|\mathscr{L}(\Gamma)|-m}{m} \leqslant c
$$

следует, что $|\mathscr{L}(\Gamma)| \leqslant m(c+1)$. С другой стороны, $|\mathscr{L}(\Gamma)| \geqslant m$.

Возможны три случая.

Пусть $m=2,3,4$. Тогда для любого $c$

$$
M_{c}(m)=M_{0}(m)=Q(m) .
$$

Пусть $2^{k}+1 \leqslant m \leqslant 2^{k}+2^{k-2}, k \geqslant 2$. Согласно лемме 5

$$
M_{0}\left(2^{k}+2^{k-2}\right)=2 k+1 \text {. }
$$

Из той же леммы следует, что при $r \geqslant 1$

$$
M_{0}\left(2^{k}+r\right) \geqslant 2 k+1 \text {. }
$$

Поэтому при $c \geqslant 1 / 4$

$$
\begin{aligned}
m(c+1) & \geqslant(2 k+1)(c+1) \geqslant\left(2^{k}+1\right)(1+1 / 4) \\
& >2^{k}(1+1 / 4)=2^{k}+2^{k-2} .
\end{aligned}
$$

Следовательно, $M_{c}(m)=2 k+1$ при $c \geqslant 1 / 4$.

Наконец, пусть $2^{k}+2^{k-2}+1 \leqslant m \leqslant 2^{k+1}, k \geqslant 2$. Согласно лемме 5 при $r \geqslant 1$

$$
M_{0}\left(2^{k+1}\right)=2(k+1)=2 k+2 \text {. }
$$

Из той же леммы следует, что

$$
M_{0}\left(2^{k}+2^{k-r}+r\right) \geqslant 2 k+2 .
$$

Поэтому при $c \geqslant 3 / 5$

$$
m(c+1) \geqslant\left(2^{k}+2^{k-r}+1\right) \frac{8}{5}=2^{k+1}+\frac{8}{5}>2^{k+1} .
$$

Теорема доказана.

\section{Список литературы}

1. Аничкин С. А., Белов С. А., Протохолы информационно-вычислителъных сетей. Радио и связь, Москва, 1989.

2. Глушков В. М., Цейтлин Г. Е., Ющенко Е. Л., Алгебра. Языки. Программирование. Наукова думка, Киев, 1978. 